\title{
Genome-Wide Association Study of Clinical Outcome After Aneurysmal Subarachnoid Haemorrhage: Protocol
}

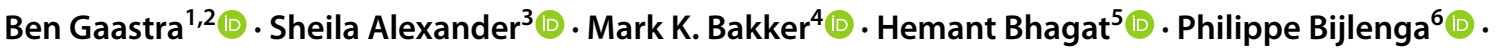

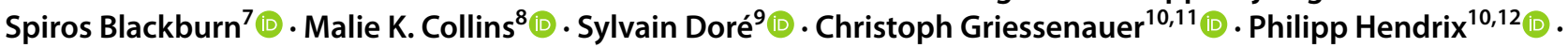 \\ Eun Pyo Hong ${ }^{13}$ (1) $\cdot$ Isabel C. Hostettler ${ }^{14}$ (D) Henry Houlden ${ }^{14} \cdot$ Koji llhara $^{15}$ (i) $\cdot$ Jin Pyeong Jeon ${ }^{13,16}$ (D)

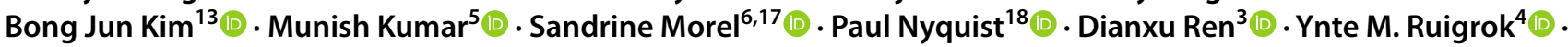

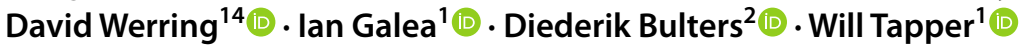

Received: 16 August 2021 / Revised: 26 November 2021 / Accepted: 13 December 2021 / Published online: 6 January 2022 (c) The Author(s) 2021

\begin{abstract}
Aneurysmal subarachnoid haemorrhage (aSAH) results in persistent clinical deficits which prevent survivors from returning to normal daily functioning. Only a small fraction of the variation in clinical outcome following aSAH is explained by known clinical, demographic and imaging variables; meaning additional unknown factors must play a key role in clinical outcome. There is a growing body of evidence that genetic variation is important in determining outcome following aSAH. Understanding genetic determinants of outcome will help to improve prognostic modelling, stratify patients in clinical trials and target novel strategies to treat this devastating disease. This protocol details a two-stage genome-wide association study to identify susceptibility loci for clinical outcome after aSAH using individual patient-level data from multiple international cohorts. Clinical outcome will be assessed using the modified Rankin Scale or Glasgow Outcome Scale at 1-24 months. The stage 1 discovery will involve meta-analysis of individual-level genotypes from different cohorts, controlling for key covariates. Based on statistical significance, supplemented by biological relevance, top single nucleotide polymorphisms will be selected for replication at stage 2 . The study has national and local ethical approval. The results of this study will be rapidly communicated to clinicians, researchers and patients through open-access publication(s), presentation(s) at international conferences and via our patient and public network.
\end{abstract}

Keywords Subarachnoid haemorrhage $\cdot$ Stroke $\cdot$ Outcome assessment $\cdot$ Health care $\cdot$ Genetics $\cdot$ Medical

\section{Introduction}

Aneurysmal subarachnoid haemorrhage (aSAH) has a severe socioeconomic burden [1] as it affects people of young age and survivors suffer persisting physical, cognitive, auditory and psychosocial morbidity [2], leading to unemployment [3]. It is a striking clinical observation that individuals with similar bleeds, clinical characteristics and comorbidities experience widely different outcomes. Dozens of modelling studies, including the most recent enrolling over 10,000

Ian Galea, Diederik Bulters and Will Tapper are joint senior authors.

Ian Galea

i.galea@soton.ac.uk

Extended author information available on the last page of the article patients [4], have consistently found that only up to a third of the variance in clinical outcome can be explained using a combination of demographic, clinical and imaging characteristics. Consequently, unknown additional factors play a key role in clinical outcome.

The mechanism of neurological injury following aSAH can be divided into an early brain injury (EBI) occurring within $72 \mathrm{~h}$ of ictus and a delayed brain injury occurring in the subsequent days to weeks after haemorrhage [5]. EBI is caused by a rapid rise in intracranial pressure and concomitant fall in cerebral blood flow at the time of haemorrhage [6]. Toxic cascades initiated by EBI and the presence of blood and its breakdown products within the cerebrospinal fluid are thought to lead to delayed brain injury, characterised by a range of pathological processes including cerebral vasospasm, inflammation, oxidative damage and cortical spreading depression [7-9]. However, the relative 
significance of each of these pathological pathways is currently unknown, impairing the development of pharmacological and therapeutic strategies to prevent or reduce neurological injury.

Previous studies have indicated that clinical outcome may be influenced by the genetic background of individual patients [10]. For example, we have shown that haptoglobin genotype influences clinical outcome [11] and others have implicated endothelial nitric oxide synthase [12], apolipoprotein E [13], brain-derived neurotrophic factor [14] and genes associated with fibrinolysis [15] and inflammation [16]. An automated search of the literature using GLAD4U, a PubMed gene retrieval and prioritisation tool, identified 324 genes associated with aSAH outcome [17, 18]. These genes were derived from candidate gene studies that rely on a priori knowledge of the genes to make the link with clinical outcome. However, as our understanding of the molecular mechanisms underlying outcome after aSAH is incomplete, these targeted approaches may overlook significant genes and are unlikely to deliver novel findings. A systematic genome-wide analysis will overcome this limitation. While there have been several genome-wide association studies (GWAS) comparing patients with and without aneurysms, or comparing patients with ruptured versus unruptured aneurysms, a GWAS of clinical outcome after aSAH has never been performed to date [19, 20]. This is primarily because of the logistic difficulties associated with collecting clinical outcome in a large number of patients when aSAH has a relatively low incidence of around 6 per 100,000 person-years [21]. In order to address the challenge of adequate case ascertainment, one method is to perform an individual patient level data analysis of retrospective data from multiple collaborators [22].

A greater understanding of the genetic variants associated with outcome following aSAH will provide valuable insight into the pathophysiological mechanisms of outcome after aSAH highlighting the pathways which underlie neurological injury, with the potential to improve patient care. Moreover, genetic variants could be used to improve current prognostic models identifying patients at risk of deterioration who may benefit from increased observation, early intervention or access to rehabilitation [23]. Improved prognostication will also allow patients and carers to scale their expectations and forward plan their work and personal life. Additionally, knowledge of genetic variants associated with clinical outcome could be used to adjust for patient heterogeneity in aSAH clinical trials allowing for patient stratification and decreasing required sample sizes [24]. Finally, genome-wide analysis of genetic variation associated with outcome may highlight genes and pathways that were previously not considered pharmaceutical targets to improve outcome. This will allow drug target analysis for the development of novel and/or repositioning of known therapies to mitigate the devastating consequences of this condition [25]. New treatments are desperately needed in aSAH as at present there is only a single drug (nimodipine) to improve outcome, the effect of which is modest [26, 27].

In this study protocol, we detail the methodology for the first GWAS of clinical outcome following aSAH. Our primary aim is to identify genetic variants which influence clinical outcome, irrespective of whether this is via a direct or indirect path. Consequently, genetic variants associated with outcome identified in this study may not directly influence outcome but rather mediate their effect through the pathological processes of EBI and delayed brain injury. We estimate the study power and describe the statistical methods, including quality control and adjustment for necessary covariates. This study will entail a major international collaboration to identify genes associated with clinical outcome following aSAH. This study will have three main impacts: (1) the development of improved prognostic models for aSAH outcome; (2) the identification of novel therapeutic targets; and (3) the building of the foundations of knowledge needed for future studies on clinical outcome after aSAH.

\section{Materials and Methods}

This study will be reported in accordance with the "STrengthening the REporting of Genetic Association studies" (STREGA) statement [28]. This study was initially conceived in 2019, with patient recruitment commencing in January 2021. The authorship includes a statistician (DR) who has advised on study design and analysis.

\section{Study Design}

International two-stage multi-centre individual patient-level data GWAS. Case-only analysis comparing good and poor outcome aSAH individuals.

\section{Case Ascertainment Sources}

Cases for inclusion in this study are being identified from two sources:

\section{Haemoglobin after intracranial haemorrhage (HATCH) consortium}

The HATCH consortium is an international consortium with a focus on outcome following brain haemorrhage, including members from Asia, the Americas and Europe. The consortium is identifying adult aSAH patients by contacting investigators worldwide, identified through clinical trial registries and PubMed searches. 
A trial registry search was performed (Table 1) using search conditions: "subarachnoid h(a)emorrhage" AND registration of trial in the last 10 years. The principal investigators were emailed in their native language, inviting them to participate in the study. In January 2019, 498 studies were identified, which were manually screened for relevance to biosample and/or genetic data availability, resulting in 148 contacts who were emailed. This search will be repeated prior to commencing the stage 2 validation analysis (see below) to ensure all available cases are identified.

In collaboration with the International Stroke Genetics Consortium (ISGC), further study sites are being identified through peer networks and presentations at ISGC workshops. In addition, an international campaign, including online advertisements and multiple oral and poster presentations at several international workshops and conferences, has also been commenced.

\section{The UK Biobank}

The UK Biobank is an ongoing population-based cohort study that aims to improve the prevention, diagnosis and treatment of a wide range of diseases. Extensive genetic and clinical data have been collected on around half a million participants across the UK that were aged between 40 and 69 at the time of recruitment from 2006 to 2010 . The design, data collection and processing are described in detail elsewhere [29]. The UK Biobank includes a substantial cohort of aSAH patients with follow-up data. The UK Biobank has approved this project proposal under application ID 49305.

\section{Inclusion/Exclusion Criteria}

\section{HATCH dataset}

Adult ( $\geq 18$ years) aSAH cases with aneurysmal cause of bleed confirmed by any angiographic method and genomewide genotype information available will be eligible for inclusion. Individuals will be excluded if no aneurysm can be identified or if a non-aneurysmal cause for subarachnoid haemorrhage, including vascular malformation and trauma, is present.

\section{UK Biobank dataset}

aSAH cases will be identified from the UK Biobank using the following data fields (Supplementary Table 1):

- ICD-9 (data field 41271) and ICD-10 (data field 41270) codes from hospital inpatient data

- Read code information from primary care data (data field 42040)

- Self-reported medical conditions (data field 20002) reported at baseline or subsequent assessment centre visits

Cases identified from the UK Biobank will be crosschecked against the algorithmically generated subarachnoid haemorrhage diagnosis (data field 42012) and first occurrence database for ICD-10 code I60 (data field 131360). Genotyped aSAH cases will be included if they have outcome data available subsequent to the date of diagnosis. Cases will be excluded if there is evidence that subarachnoid haemorrhage is secondary to non-aneurysmal pathologies such as vascular malformation or trauma. Non-aneurysmal causes for subarachnoid haemorrhage will be identified using ICD-9 and ICD-10 codes indicative of non-aneurysmal SAH from hospital inpatient and primary care data (Supplementary Table 1) and individuals with such a code will be excluded regardless of the time interval between diagnosis of subarachnoid haemorrhage and potential non-aneurysmal cause. With respect to traumatic event codes, cases will only be excluded if the date of these events indicates that trauma occurred within 30 days before or after the diagnosis of subarachnoid haemorrhage.

\section{Primary Outcomes and Covariates}

\section{HATCH dataset}

The primary outcome will be dichotomised clinical outcome (assessed at 1-24 months), based on the modified Rankin Scale (mRS) [30-32] or Glasgow Outcome Scale (GOS) [33, 34], which correlate highly with each other $\left(R_{\mathrm{S}}\right.$ $=-0.90, p<0.001$, manuscript under review). Outcome will be dichotomised into favourable $(\mathrm{mRS}=0-2, \mathrm{GOS}=4-5)$ and
Table 1 Table of trial registries searched to identify cases for inclusion in the study

\begin{tabular}{ll}
\hline Trial registry & Website \\
\hline WHO International Clinical Trials Registry Platform & http://apps.who.int/trialsearch/ \\
ClinicalTrials.gov & https://clinicaltrials.gov/ \\
European Clinical Trials Database & $\mathrm{https} / /$ www.clinicaltrialsregister.eu/ctr-search/ \\
& search \\
International Standard Registered Clinical/Social Study & http://www.isrctn.com/ \\
Number (ISRCTN) registry & \\
\hline
\end{tabular}


unfavourable ( $\mathrm{mRS}=3-6, \mathrm{GOS}=1-3)$, enabling both scales to be used [4]. If both mRS and GOS data are available from a single study only, the variable with the greatest data availability will be used, i.e. mixed mRS or GOS data from individual study sites will not be used.

\section{UK Biobank dataset}

The primary outcomes will be employment status (data field 6142) and cognition, as measured by reaction time (data field 20023) following aSAH. These measures have been chosen as they have been shown to detect differences in outcome between aSAH cases and controls within the UK Biobank cohort [35].

Employment status will be dichotomised into good and poor outcome with poor outcome defined as "unable to work because of sickness or disability" or "unemployed". Reaction times will be ranked and then dichotomised so that the lower scoring poor outcome group is equivalent, in terms of percentage of the total UK Biobank aSAH cohort, to the poor outcome group in the HATCH dataset. Cognitive outcome and mRS after aSAH are highly correlated [36].

\section{Essential Covariates}

The primary aim is not to explain maximum variance as is conventionally done in predictive modelling but to detect associations between genetic variation and outcome. We have limited covariates to confounding variables since this is essential in establishing causality; confounding variables are defined by a forward path linking the variable to both exposure and outcome. Directed acyclic graph theory has been used to rationalise the choice of covariates. Age and genetic ancestry $[37,38]$ are the only known variables satisfying this definition and will be included as essential covariates (Fig. 1A).

Population stratification will be assessed by principal component analysis, using reference populations from the 1000 Genomes Project [39], and the top five genetic ancestry eigenvectors will be used as covariates.

\section{Additional Covariates}

In addition to age and genetic ancestry, a number of variables have been shown to predict outcome following aSAH. These predictors include baseline characteristics such as the World Federation of Neurological Surgeons (WFNS) score [4] and features of the patient's clinical course, for example, aneurysm treatment modality [40], rebleed and delayed cerebral ischemia (DCI) [41]. As these variables are expected to influence outcome but not an individual's genetic profile, they are not considered as confounding variables (Fig. 1B). However, one or more of these variables may mediate a
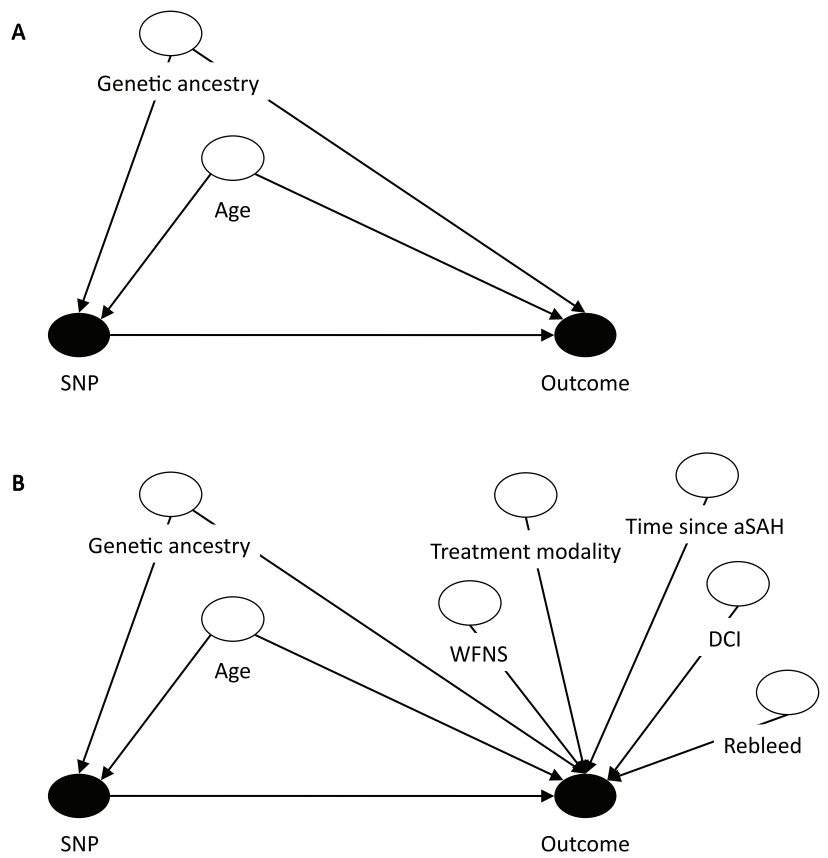

C

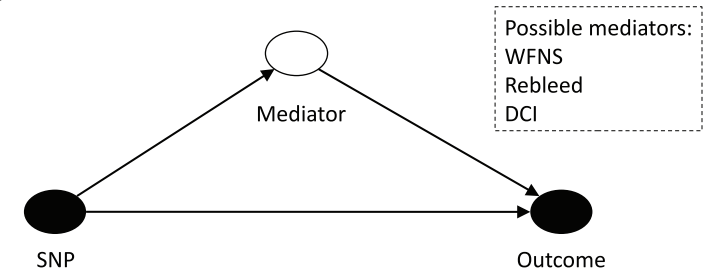

Fig. 1 A Directed acyclic graph (DAG) demonstrating confounding covariate interaction with exposure (SNP) and outcome - primary analysis. B DAG demonstrating both confounding and selected nonconfounding covariates - predictive modelling in cases with available data, for genetic variants confirmed in A. C Pathway diagram demonstrating possible mediation of a gene $\rightarrow$ outcome effect by WFNS, rebleed or DCI, for genetic variants confirmed in A. SNP, single nucleotide polymorphism; WFNS, World Federation of Neurological Surgeons; aSAH, aneurysmal subarachnoid haemorrhage; DCI, delayed cerebral ischemia

proportion of the effect of genetic variation on outcome, and hence can be viewed as "mechanistic" variables linking gene to outcome. This is more likely for mechanistic variables with proven links to outcome such as DCI and aneurysm rebleed, both of which also happen to have a proposed genetic component $[10,19]$. By focussing on the gene $\rightarrow$ outcome pathway irrespective of mediating variables, this study will detect the genes that matter as potential biological targets for new treatments. In addition, this approach has the added advantage of maximising sample size since it is not dependent on the availability of additional covariates.

As an exploratory endpoint where data availability allows, regression-based mediation analysis will be used to explore whether potential mechanistic variables (WFNS score or Hunt and Hess $(\mathrm{H} \& \mathrm{H})$ grade, aneurysm rebleed 
and presence of DCI) mediate any proportion of the genetic effect on outcome following aSAH, for genetic variants confirmed in the primary analysis (Fig. 1C). If data availability allows, an attempt will be made to construct a predictive model to explain maximum variance in outcome, including the covariates: genetic variants confirmed in the primary analysis, age, genetic ancestry, time since aSAH, WFNS score or $\mathrm{H} \& \mathrm{H}$ grade, treatment modality categorised as conservative, endovascular and surgical, aneurysm rebleed and presence of DCI (Fig. 1B). This will be performed as a sensitivity analysis as data is not available for all samples.

In this study, clinical outcome in the HATCH cohort is assessed over a 1- to 24-month time period. This range is broad to maximise patient inclusion in the study. The UK Biobank time to follow-up after aSAH is also broad (1 to 662 months). We have not restricted the time period over which outcome can be assessed in the UK Biobank as we have shown that cognitive and employment outcomes differ between cases and controls over this time period [35], allowing maximum patient inclusion in the study. Outcome is expected to be associated with time since aSAH (Fig. 1B) [42]. Hence, time to follow-up will be included as an additional covariate in a sensitivity analysis of significant genetic variants identified in the primary analysis. We will also conduct a sensitivity analysis including only individuals with follow-up at 3 to 6 months.

In the UK Biobank WFNS is not available, so the length of stay will be used instead since this has a strong association with WFNS [43]. As length of stay data has high missingness (around 40\%) and as it is a surrogate of the strongest predictor of clinical outcome [4], it will be imputed using a method of mean imputation to allow for inclusion in the analysis. As the UK Biobank cohort uses cognitive performance and employment as surrogate measures of outcome, the additional covariates Townsend deprivation score [44] (data field 189) and education status dichotomised into individuals holding a college or university degree at the time of initial assessment in the UK Biobank or not (data field 6138) will be included in a sensitivity analysis. For the reaction time analysis, the presence of medications known to influence reaction time in the UK Biobank [45] will also be used.

\section{Genotype Quality Control}

Where possible, single nucleotide polymorphism (SNP) data will be sought from collaborators with genotype calls relative to the positive strand. Datasets that are not genotyped on the positive strand will be identified and flipped to the positive strand using SNPFLIP. Genome-wide genotype data will be subjected to standard quality control methods. Patients with gender mismatch, individual missingness $>10 \%$, heterozygosity rates \pm 3 standard deviations from the samples' heterozygosity rate mean and cryptic relatedness (proportional identity by descent $>0.1875$ ) will be excluded. SNPs with extreme deviation from Hardy-Weinberg equilibrium, minor allele frequency (MAF) of $<1 \%$ and SNP call rate $<90 \%$ will be excluded. In preparation for imputation and to resolve any residual strand issues, SNPs will be excluded if they are absent from the haplotype reference consortium (HRC), their alleles disagree with HRC, their MAF differ by greater than 0.2 versus HRC or they are palindromic and have MAF greater than 0.4.

\section{Imputation}

In the HATCH dataset, imputation will be needed since the genetic data has been obtained on different platforms; it will also increase the density of coverage to enable fine mapping around significant loci. If data has already been imputed by the contributing study teams, this imputation will be used; otherwise, imputation will be performed using the Sanger Imputation Service [46]. Haplotypes will be pre-phased using EAGLE2 [47] into the Haplotype Reference Consortium (r1.1) [46] and imputed using the positional BurrowsWheeler transform [48]. Imputed genotypes will be quality controlled by excluding SNPs with a posterior genotype probability less than 0.8 , a MAF less than $5 \%$, greater than $10 \%$ missing genotypes within the cohort or extreme deviation from Hardy-Weinberg equilibrium $\left(p \leq 1 \times 10^{-10}\right)$.

For datasets already quality controlled and imputed by the contributing team, the above metrics will be reapplied to ensure harmonisation with the exception of heterozygosity which relies on standard deviation of the mean and will therefore be manually reviewed, and imputation quality for which we will report the threshold for each imputed dataset.

\section{Data Analysis}

The GWAS will be performed in two stages: discovery and validation (Fig. 2).

\section{Stage 1: Discovery}

In the first stage, available cohorts from the HATCH consortium will be analysed separately. Genetic variants will be tested for association using multivariable logistic regression analyses with dichotomised clinical outcome as dependent, genetic variant as predictor, and including the essential covariates specified for the analysis, as detailed above. Residual population stratification will be monitored using lambda from QQ plots and corrected if required. Fixed effects inverse variance weighted meta-analysis of the individual studies will be performed to determine the overall significance and effect size of individual genetic variants in the HATCH consortium. Finally, heterogeneity between cohorts will be examined using Cochrane's $Q$ and $I^{2}$ statistics. 
Fig. 2 Analysis plan based on two stages: discovery and validation

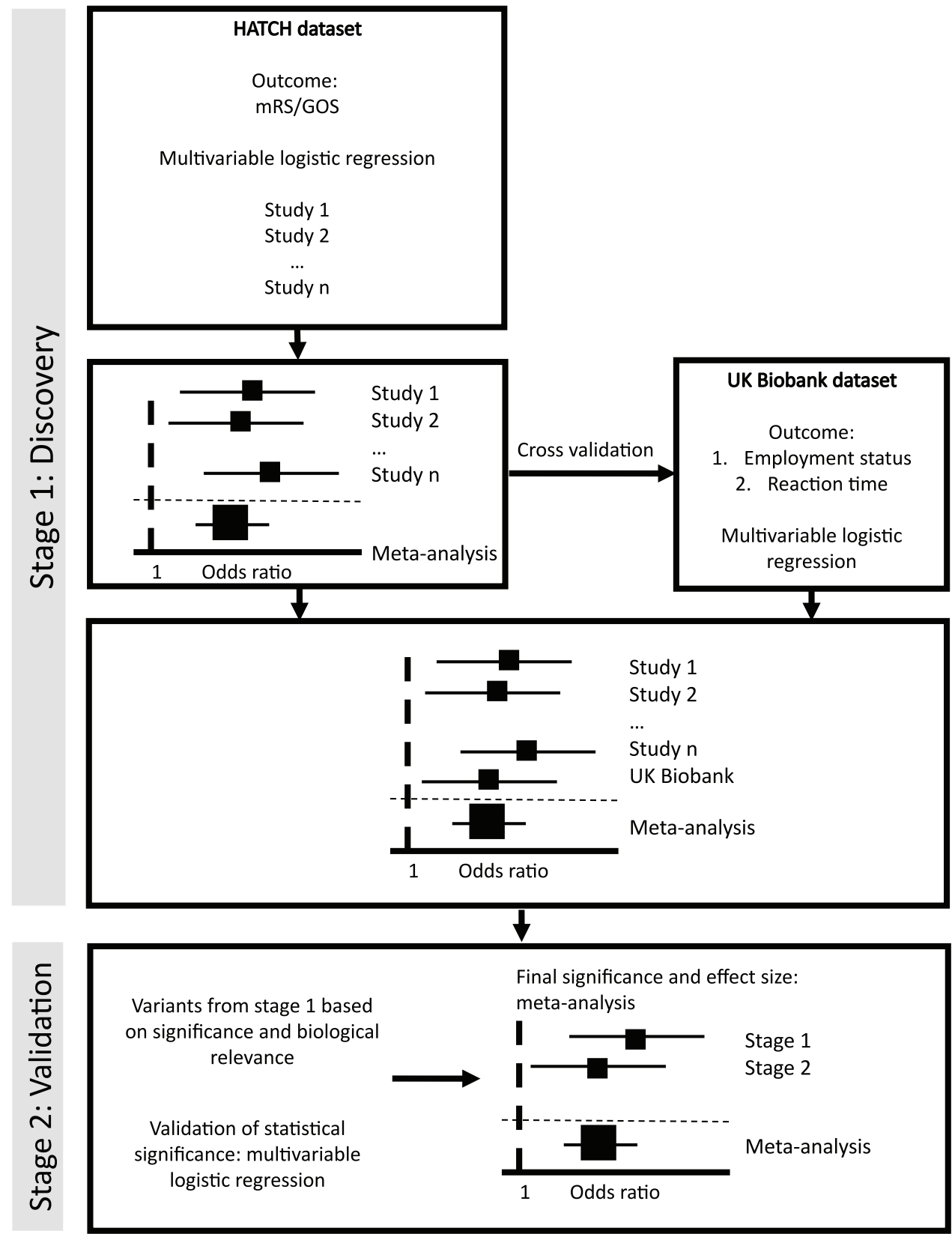

The UK Biobank will be considered a single study site and undergo the same multivariable logistic regression analysis as the HATCH dataset. Within the UK Biobank dataset, dichotomised employment status and reaction time outcomes will be considered as separate analyses. Significant findings within the HATCH dataset will be cross-validated in the UK Biobank cohort. As cognitive and traditional (mRS/GOS) outcome measures are correlated after SAH [36], a metaanalysis of summary statistics from both $\mathrm{HATCH}$ and UK Biobank datasets will also be performed as the final output of the stage 1 discovery analysis.

To explore the functional relevance of regions associated with clinical outcome, FUMA [49] will be used to determine if the risk SNPs and their proxies $\left(r^{2} \geq 0.6\right.$, within $1 \mathrm{Mb}$ and nominally significant) are located within putative functional elements such as active histone marks or transcription factor binding motifs. Additionally, FUMA will be used to annotate these SNPs with respect to evidence of regulatory function using Regulome DB scores [50], combined annotation-dependent depletion scores [51] and gene expression using eQTL analysis [52]. Furthermore, genes functionally related to the risk SNPs will be explored as drug targets using individual gene and network-based approaches. Finally, phenomewide association study techniques will be used to identify SNPs with pleiotropic effects. 


\section{Stage 2: Validation}

Genetic variants with the greatest significance in stage 1 will be identified for replication in the stage 2 validation analysis. Variants from stage 1 will be selected for stage 2 based on statistical significance, with all variants with $p<1 \times 10^{-4}$ considered for replication. The top variants, as ranked by $p$-value, will be included in the validation study. In addition, to maximise the identification of replicable variants in stage 2 [53], variants from stage 1 with $p<1 \times 10^{-4}$ and evidence of biological/functional relevance will also be included in the validation study. The validation study will use the same multivariable logistic regression analysis as stage 1. Only variants that replicate in the validation study will be considered to be truly associated with outcome with the final significance and effect size determined by a fixed effects meta-analysis of stages 1 and 2 .

\section{Sensitivity Analyses}

In order to account for time since aSAH, it will be included as an additional covariate with significant genetic variants from the primary analysis retested, incorporating this variable to ensure an independent genetic effect (Fig. 1B). In addition, a further sensitivity analysis will be performed, including only individuals with follow-up at 3 to 6 months.

As the UK Biobank cohort uses employment and cognition as surrogate measures of outcome, a sensitivity analysis including the additional covariates described above will be performed to ensure independent genetic effect. Finally, the UK Biobank cohort will be excluded to ensure no change to the significance of the results.

\section{Mediation Analyses}

Regression-based mediation analysis will be used to explore whether potential mechanistic variables (WFNS score or $\mathrm{H} \& \mathrm{H}$ grade, aneurysm rebleed and presence of DCI) mediate any proportion of the genetic effect on outcome following aSAH, for genetic variants confirmed in the primary analysis.

\section{Sample Size and Power Calculation}

A recent study demonstrated that in high Fisher grade (III-IV) individuals, the haptoglobin 2-2 genotype was associated with good clinical outcome (mRS 0-1) following aSAH with an odds ratio of 2.6 (95\% confidence interval 1.4-4.9) [11]. Based on this finding, we aim to power this study to detect common genetic variation with an effect size of $>1.4$, the lower end of the $95 \%$ confidence interval. After aSAH, $30 \%$ are expected to have an unfavourable outcome [54].
At present, approximately 2500 retrospective samples have been identified for inclusion in the stage 1 (discovery) analysis. See www.southampton.ac.uk/hatch/studies/ gwas.page for a live tally of sample number and study sites. Recruitment is ongoing for the stage 2 (validation) study with multiple international collaborators. The co-authors of this study have either provided data for stage 1 or are providing samples or data for the stage 2 analysis.

Based on the current stage 1 discovery analysis sample size of 2500 , according to the above event rate and assuming an additive model, the stage 1 analysis will have $80 \%$ power to detect common SNPs (MAF=0.4) with an effect size of 1.48 and rare SNPs $(\mathrm{MAF}=0.1)$ with an effect size of 1.86 at a genome-wide level of significance (Fig. 3A). In the final meta-analysis combining stage 1 and stage 2 , a sample size of 5000 is predicted, which using the same assumptions will have $80 \%$ power to detect common SNPs $(\mathrm{MAF}=0.4)$ with an effect size of 1.33 and rare SNPs $(\mathrm{MAF}=0.1)$ with an effect size of 1.56 at a genome-wide level of significance (Fig. 3B).

\section{Limitations}

This study does not include individuals who died prior to hospital admission. In addition, it is possible that recruitment is biased towards better outcome individuals as poor prognosis individuals who die in the first few days after aSAH are less likely to be recruited. We will assess this bias by comparing the proportion of good/poor outcome patients in this study with that in contemporary observational studies of outcome and case fatality [55]. Even if such a bias is identified, it will not be a significant limitation to this study as the ultimate aim is to identify pathophysiological mechanisms which can act as therapeutic targets to improve outcome. After aSAH, the time window over which individuals deteriorate following a bleed is relatively long (days to weeks) and during this time patients usually remain in hospital. This means that there is a window of opportunity to administer treatments to prevent deterioration and poor outcome following haemorrhage. Unfortunately, individuals who die prior to admission or in the first few days after aSAH are unlikely to benefit from such interventions. This means that although our study population may be biased away from individuals who die in the first few days after aSAH, it includes the individuals who realistically will benefit from the output of this study.

The UK Biobank has different outcome measures (cognition and employment) than the HATCH datasets that employ the mRS or GOS. This limits the comparability of the UK Biobank and HATCH datasets. To address this limitation, we have separated the UK Biobank and HATCH datasets, and the UK Biobank will be used to cross-validate findings from the HATCH datasets. This allows significant findings 
Fig. 3 Graph of power versus SNP effect size for a range of minor allele frequencies at genome-wide significance. Dashed line identifies effect size at $80 \%$ power. A Sample size $=$ 2500 (stage 1); $\mathbf{B}$ sample size $=$ 5000 (final meta-analysis: stage $1+$ stage 2)
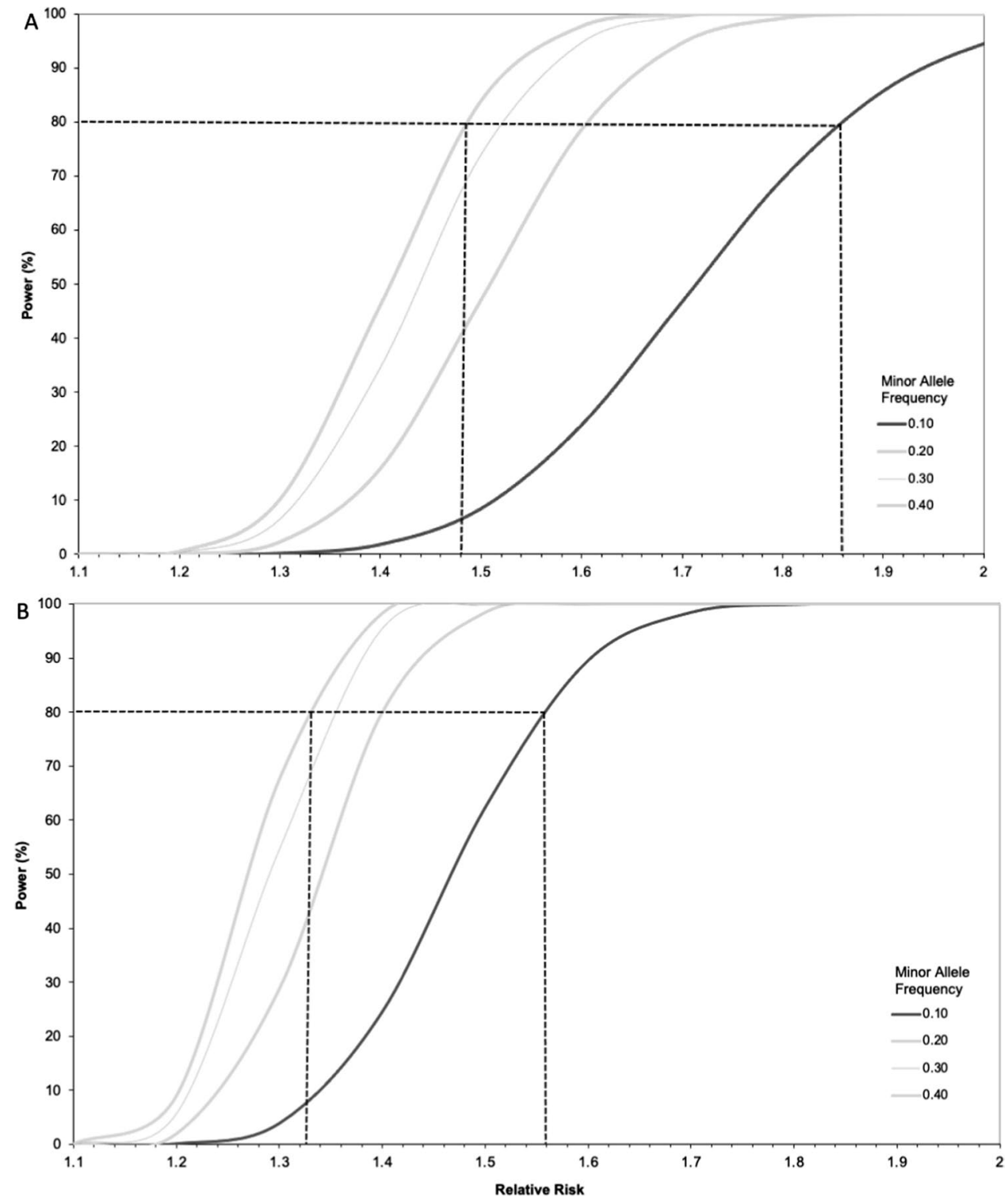

in the HATCH data to be further validated using outcome metrics more sensitive to the nuances of outcome and more relevant to patients than $\mathrm{mRS} / \mathrm{GOS}$. We also combine the UK Biobank and HATCH datasets to maximise study power. Within the UK Biobank, only $2 \%$ of individuals have psychomotor reaction time and/or employment status recorded both before and after aSAH. Nevertheless, a detailed analysis of employment and cognition in the UK Biobank has shown that psychomotor reaction time and employment are significantly impaired compared to matched controls and therefore constitute valid outcome measures [35].

\section{Patients and Public Involvement}

We have worked with the Wessex Subarachnoid Haemorrhage Support Group and participants from previous research studies of patients with aSAH to prioritise research questions important to them and their carers since 2012, holding regular meetings and workshops. The group identified maximising use of samples obtained from prior studies as an important principle and understanding mechanisms of poor outcome to develop new treatments as a priority.

\section{Ethics}

For this study, national (REC 19 SC 0485) and local (ERGO 49253) ethical approvals are already in place.

\section{Dissemination}

The output of this discovery study will be published in relevant open-access peer-reviewed journals to ensure rapid 
dissemination to the target audience. All contributors to the study will be co-authors on manuscripts alphabetised between first and senior authors. Results of the study will also be presented at national and international stroke and aSAH meetings. In addition, through our links with the Wessex SAH support group, we will promote the output of this study to patients and the public along with presentation of results on the HATCH, local hospital and university websites, and social media.

Supplementary Information The online version contains supplementary material available at https://doi.org/10.1007/s12975-021-00978-2.

Code Availability Not applicable

Authors Contribution All authors contributed to the study's conception and design. The first draft of the manuscript was written by BG, WT, IG and DB; all authors commented on previous versions of the manuscript. Finally, all authors read and approved the final manuscript.

Funding BG is funded by the Royal College of Surgeons, Society of British Neurological Surgeons and Barrow Foundation, and the Institute for Life Sciences, University of Southampton. DJW, HH and IH received funding for recruitment to the GOSH study and genotyping from the Stroke Association and the UCLH NIHR Biomedical Research Centre. SA received funding for data and sample collection from the National Institute of Nursing Research (R01NR004339). YR received funding from the Netherlands Cardiovascular Research Initiative: An initiative with the support of the Dutch Heart Foundation, CVON2015-08 ERASE, and from the European Research Council (ERC) under the European Union's Horizon 2020 research and innovation programme (PRYSM, grant agreement No. 852173). PB and SM received funding for the AneuX project from SystemsX.ch, which was evaluated by the Swiss National Science Foundation. SB is supported by NIH Grant (K23NS106054). SD is funded by the National Institute of Neurological Disorders and Stroke (1R56NS11607601A1 \& 1R21NA110008-01A1) and The Assistant Secretary of Defense for Health Affairs endorsed by the Department of Defense (W81XWH1910606)

Data Availability Study data will be available from the authors subject to institutional agreements and ethical approvals.

\section{Declarations}

Ethics Approvals National (REC 19 SC 0485) and local (ERGO 49253) ethical approval in place.

Consent to Participate Informed consent was obtained from all individual participants included in the study.

\section{Consent for Publication Not applicable}

Conflict of Interest The authors declare no competing interests.

Open Access This article is licensed under a Creative Commons Attribution 4.0 International License, which permits use, sharing, adaptation, distribution and reproduction in any medium or format, as long as you give appropriate credit to the original author(s) and the source, provide a link to the Creative Commons licence, and indicate if changes were made. The images or other third party material in this article are included in the article's Creative Commons licence, unless indicated otherwise in a credit line to the material. If material is not included in the article's Creative Commons licence and your intended use is not permitted by statutory regulation or exceeds the permitted use, you will need to obtain permission directly from the copyright holder. To view a copy of this licence, visit http://creativecommons.org/licenses/by/4.0/.

\section{References}

1. O. Rivero-Arias, A. Gray, and J. Wolstenholme, "Burden of disease and costs of aneurysmal subarachnoid haemorrhage (aSAH) in the United Kingdom," Cost Eff Resour Alloc, vol. 8, p. 6, Apr 27 2010, 10.1186/1478-7547-8-6.

2. A. Pace et al., "A subarachnoid haemorrhage-specific outcome tool," Brain, vol. 141, no. 4, pp. 1111-1121, Apr 1 2018, 10.1093/brain/awy003.

3. S. Wallmark, E. Ronne-Engstrom, and E. Lundstrom, "Predicting return to work after subarachnoid hemorrhage using the Montreal Cognitive Assessment (MoCA)," Acta Neurochir (Wien), vol. 158, no. 2, pp. 233-239, Feb 2016, https://doi.org/ 10.1007/s00701-015-2665-4.

4. Jaja BNR, et al. Development and validation of outcome prediction models for aneurysmal subarachnoid haemorrhage: the SAHIT multinational cohort study. BMJ. 360:j5745. https://doi. org/10.1136/bmj.j5745.

5. D. Bulters et al., "Haemoglobin scavenging in intracranial bleeding: biology and clinical implications," Nat Rev Neurol, vol. 14, no. 7, pp. 416-432, Jul 2018, https://doi.org/10.1038/ s41582-018-0020-0.

6. Cahill J, Cahill WJ, Calvert JW, Calvert JH, Zhang JH. Mechanisms of early brain injury after subarachnoid hemorrhage. $J$ Cereb Blood Flow Metab. 2006;26(11):1341-53. https://doi.org/ 10.1038/sj.jcbfm.9600283.

7. Zolnourian A, Galea I, Bulters D. Neuroprotective role of the Nrf2 pathway in subarachnoid haemorrhage and its therapeutic potential. Oxid Med Cell Longev. 2019;2019:6218239. https:// doi.org/10.1155/2019/6218239.

8. S. D'Souza, "Aneurysmal subarachnoid hemorrhage," J Neurosurg Anesthesiol, vol. 27, no. 3, pp. 222-240, Jul 2015, https:// doi.org/10.1097/ANA.0000000000000130.

9. R. L. Macdonald, R. M. Pluta, and J. H. Zhang, "Cerebral vasospasm after subarachnoid hemorrhage: the emerging revolution," Nat Clin Pract Neurol, vol. 3, no. 5, pp. 256-263, May 2007, https://doi.org/10.1038/ncpneuro0490.

10. A. F. Ducruet et al., "Genetic determinants of cerebral vasospasm, delayed cerebral ischemia, and outcome after aneurysmal subarachnoid hemorrhage," J Cereb Blood Flow Metab, vol. 30, no. 4, pp. 676-688, Apr 2010, https://doi.org/10.1038/jcbfm. 2009.278 .

11. Morton MJ, et al. Haptoglobin genotype and outcome after aneurysmal subarachnoid haemorrhage. J Neurol Neurosurg Psychiatry. 91(3):305-13. https://doi.org/10.1136/ jnnp-2019-321697.

12. R. M. Starke et al., "Endothelial nitric oxide synthase gene single-nucleotide polymorphism predicts cerebral vasospasm after aneurysmal subarachnoid hemorrhage," J Cereb Blood Flow Metab, vol. 28, no. 6, pp. 1204-1211, Jun 2008, https://doi.org/ $10.1038 / \mathrm{jcbfm} .2008 .11$.

13. M. J. Gallek, Y. P. Conley, P. R. Sherwood, M. B. Horowitz, A. Kassam, and S. A. Alexander, "APOE genotype and functional outcome following aneurysmal subarachnoid hemorrhage," Biol Res Nurs, vol. 10, no. 3, pp. 205-212, Jan 2009, https://doi.org/ $10.1177 / 1099800408323221$ 
14. Vilkki J, Lappalainen J, Juvela S, Kanarek K, Hernesniemi JA, Siironen J. Relationship of the Met allele of the brain-derived neurotrophic factor Val66Met polymorphism to memory after aneurysmal subarachnoid hemorrhage. Neurosurgery. 2008;63(2):198-203; discussion 203. https://doi.org/10.1227/ 01.NEU.0000320382.21577.8E

15. M. D. Vergouwen, C. J. Frijns, Y. B. Roos, G. J. Rinkel, F. Baas, and M. Vermeulen, "Plasminogen activator inhibitor-1 $4 \mathrm{G}$ allele in the $4 \mathrm{G} / 5 \mathrm{G}$ promoter polymorphism increases the occurrence of cerebral ischemia after aneurysmal subarachnoid hemorrhage," Stroke, vol. 35, no. 6, pp. 1280-1283, Jun 2004, https://doi.org/10.1161/01.STR.0000128707.48644.7e.

16. Y. M. Ruigrok, A. J. Slooter, A. Bardoel, C. J. Frijns, G. J. Rinkel, and C. Wijmenga, "Genes and outcome after aneurysmal subarachnoid haemorrhage," J Neurol, vol. 252, no. 4, pp. 417-422, Apr 2005, https://doi.org/10.1007/s00415-005-0661-y.

17. Jourquin J, Duncan D, Shi Z, Zhang B. GLAD4U: deriving and prioritizing gene lists from PubMed literature. $B M C$ Genomics. 2012;13(Suppl 8):S20. https://doi.org/10.1186/ 1471-2164-13-S8-S20.

18. B. Gaastra and I. Galea, "Candidate molecular predictors of outcome after aneurysmal subarachnoid haemorrhage: a systematic review of haemoglobin metabolism, inflammation and oxidative injury pathways," medRxiv, p. 19004853, 2019, https://doi.org/10. $1101 / 19004853$.

19. Bakker MK, et al. Genome-wide association study of intracranial aneurysms identifies 17 risk loci and genetic overlap with clinical risk factors. Nat Genet. 2020;52(12):1303-13. https://doi.org/10. 1038/s41588-020-00725-7.

20. Y. M. Ruigrok and G. J. Rinkel, "From GWAS to the clinic: risk factors for intracranial aneurysms," Genome Med, vol. 2, no. 9, p. 61, Sep 2010, https://doi.org/10.1186/gm182.

21. Etminan N, et al. Worldwide incidence of aneurysmal subarachnoid hemorrhage according to region, time period, blood pressure, and smoking prevalence in the population: a systematic review and meta-analysis. Jama Neurol. 2019;76(5):588-97. https://doi. org/10.1001/jamaneurol.2019.0006.

22. B. Gaastra et al., "Haptoglobin genotype and aneurysmal subarachnoid hemorrhage: individual patient data analysis," Neurology, vol. 92, no. 18, pp. e2150-e2164, Apr 2019, https://doi.org/ 10.1212/WNL.0000000000007397.

23. Lewis CM, Vassos E. Polygenic risk scores: from research tools to clinical instruments. Genome Med. 2020;12(1):44. https://doi. org/10.1186/s13073-020-00742-5.

24. E. Hughes et al., "Ancestrally unbiased polygenic breast cancer (BC) risk assessment," Journal of Clinical Oncology, vol. 39, no. 15_suppl, pp. 10502-10502, 2021, https://doi.org/10.1200/JCO. 2021.39.15_suppl.10502.

25. Cao C, Moult J. GWAS and drug targets. BMC Genomics. 2014;15(Suppl 4):S5. https://doi.org/10.1186/ 1471-2164-15-S4-S5.

26. J. D. Pickard et al., "Effect of oral nimodipine on cerebral infarction and outcome after subarachnoid haemorrhage: British aneurysm nimodipine trial," $B M J$, vol. 298, no. 6674, pp. 636-642, 1989. [Online]. Available: https://doi.org/10.1136/bmj.298.6674. 636.

27. S. M. Dorhout Mees et al., "Calcium antagonists for aneurysmal subarachnoid haemorrhage," Cochrane Database Syst Rev, no. 3, p. CD000277, 2007, https://doi.org/10.1002/14651858.CD000 277.pub3.

28. J. Little et al., "STrengthening the REporting of Genetic Association Studies (STREGA)--an extension of the STROBE statement," Genet Epidemiol, vol. 33, no. 7, pp. 581-598, Nov 2009, https:// doi.org/10.1002/gepi.20410.

29. Sudlow C, et al. UK biobank: an open access resource for identifying the causes of a wide range of complex diseases of middle and old age. PLoS Med. 2015;12(3):e1001779. https://doi.org/10. 1371/journal.pmed.1001779.

30. B. Farrell, J. Godwin, S. Richards, and C. Warlow, "The United Kingdom transient ischaemic attack (UK-TIA) aspirin trial: final results," J Neurol Neurosurg Psychiatry, vol. 54, no. 12, pp. 10441054, Dec 1991. [Online]. Available: https://doi.org/10.1136/jnnp. 54.12 .1044$.

31. T. J. Quinn, J. Dawson, M. R. Walters, and K. R. Lees, "Reliability of the modified Rankin Scale: a systematic review," Stroke, vol. 40, no. 10, pp. 3393-3395, Oct 2009, https://doi. org/10.1161/STROKEAHA.109.557256.

32. Rankin J. Cerebral vascular accidents in patients over the age of 60. II. Prognosis. Scott Med J. 1957;2(5):200-15. https://doi. org/10.1177/003693305700200504.

33. B. Jennett and M. Bond, "Assessment of outcome after severe brain damage," Lancet, vol. 1, no. 7905, pp. 480-4, 1975. [Online]. Available: https://doi.org/10.1016/S0140-6736(75) 92830-5.

34. Jennett B, Snoek J, Bond MR, Brooks N. Disability after severe head injury: observations on the use of the Glasgow Outcome Scale. J Neurol Neurosurg Psychiatry. 1981;44(4):285-93. https:// doi.org/10.1136/jnnp.44.4.285.

35. Gaastra B, Ewbank F, Tapper W, Bulters D, Galea I. Longterm cognitive outcome following aneurysmal subarachnoid haemorrhage. Journal of Stroke and Cerebrovascular Diseases. 2022;31(1):106184. https://doi.org/10.1016/j.jstrokecerebrov asdis.2021.106184.

36. Mayer SA, et al. Global and domain-specific cognitive impairment and outcome after subarachnoid hemorrhage. Neurology. 2002;59(11):1750-8. https://doi.org/10.1212/01.wnl.0000035748. 91128.c2.

37. Zacharia BE, Grobelny BT, Komotar RJ, Sander Connolly E, Mocco J. The influence of race on outcome following subarachnoid hemorrhage. J Clin Neurosci. 2010;17(1):34-7. https://doi. org/10.1016/j.jocn.2009.05.015.

38. Eden SV, et al. The role of race in time to treatment after subarachnoid hemorrhage. Neurosurgery. 2007;60(5):837-43; discussion 837-43. https://doi.org/10.1227/01.NEU.0000255451.82483.50.

39. Auton A, et al. A global reference for human genetic variation. Nature. 2015;526(7571):68-74. https://doi.org/10.1038/natur e15393.

40. Gaastra B, et al. CRP (C-reactive protein) in outcome prediction after subarachnoid hemorrhage and the role of machine learning. Stroke. 2021;52(10):3276-85. https://doi.org/10.1161/STROK EAHA.120.030950.

41. Galea JP, Dulhanty L, Patel HC, U. A. I. S. H. D. Collaborators. Predictors of outcome in aneurysmal subarachnoid hemorrhage patients: observations from a multicenter data set. Stroke. 2017;48(11):2958-63. https://doi.org/10.1161/STROKEAHA. 117.017777 .

42. Haug T, Sorteberg A, Sorteberg W, Lindegaard KF, Lundar T, Finset A. Cognitive outcome after aneurysmal subarachnoid hemorrhage: time course of recovery and relationship to clinical, radiological, and management parameters. Neurosurgery. 2007;60(4):649-56; discussion 656-7. https://doi.org/10.1227/ 01.NEU.0000255414.70807.A0.

43. Yousef K, Crago E, Fisher A, Mahmoud K, Lagattuta T, Hravnak M. Grading scales in subarachnoid hemorrhage: which scale to control for when studying outcomes. Critical Care Medicine. 2019;47(1):S2. https://doi.org/10.1097/01.ccm.0000550795. 32974.8f.

44. J. P. Mackenbach, "Health and deprivation. Inequality and the North: by P. Townsend, P. Phillimore and A. Beattie (eds.) Croom Helm Ltd, London, 1987221 pp., ISBN 0-7099-4352-0, [pound sign]8.95," Health Policy, vol. 10, no. 2, pp. 207-206, 
1988. [Online]. Available: https://doi.org/10.1016/0168-8510(88) 90006-1.

45. Nevado-Holgado AJ, Kim CH, Winchester L, Gallacher J, Lovestone S. Commonly prescribed drugs associate with cognitive function: a cross-sectional study in UK Biobank. BMJ Open. 2016;6(11):e012177. https://doi.org/10.1136/bmjop en-2016-012177.

46. McCarthy S, et al. A reference panel of 64,976 haplotypes for genotype imputation. Nat Genet. 2016;48(10):1279-83. https:// doi.org/10.1038/ng.3643.

47. Loh PR, et al. Reference-based phasing using the Haplotype Reference Consortium panel. Nat Genet. 2016;48(11):1443-8. https:// doi.org/10.1038/ng.3679.

48. Durbin R. Efficient haplotype matching and storage using the positional Burrows-Wheeler transform (PBWT). Bioinformatics. 2014;30(9):1266-72. https://doi.org/10.1093/bioinformatics/ btu014.

49. Watanabe K, Taskesen E, van Bochoven A, Posthuma D. Functional mapping and annotation of genetic associations with FUMA. Nat Commun. 1826;8(1):2017. https://doi.org/10.1038/ s41467-017-01261-5.

50. Boyle AP, et al. Annotation of functional variation in personal genomes using RegulomeDB. Genome Res. 2012;22(9):1790-7. https://doi.org/10.1101/gr.137323.112.
51. Kircher M, Witten DM, Jain P, O'Roak BJ, Cooper GM, Shendure J. A general framework for estimating the relative pathogenicity of human genetic variants. Nat Genet. 2014;46(3):310-5. https:// doi.org/10.1038/ng.2892.

52. Consortium GT. Human genomics. The Genotype-Tissue Expression (GTEx) pilot analysis: multitissue gene regulation in humans. Science. 2015;348(6235):648-60. https://doi.org/10.1126/science. 1262110.

53. Huffman JE. Examining the current standards for genetic discovery and replication in the era of mega-biobanks. Nat Commun. 2018;9(1):5054. https://doi.org/10.1038/s41467-018-07348-x.

54. Kirkpatrick PJ, Turner CL, Smith C, Hutchinson PJ, Murray GD, Collaborators S. Simvastatin in aneurysmal subarachnoid haemorrhage (STASH): a multicentre randomised phase 3 trial. Lancet Neurol. 2014;13(7):666-75. https://doi.org/10.1016/S14744422(14)70084-5.

55. Schatlo B, et al. Incidence and outcome of aneurysmal subarachnoid hemorrhage: the Swiss Study on Subarachnoid Hemorrhage (Swiss SOS). Stroke. 2021;52(1):344-7. https://doi.org/10.1161/ STROKEAHA.120.029538.

Publisher's Note Springer Nature remains neutral with regard to jurisdictional claims in published maps and institutional affiliations. 


\section{Authors and Affiliations}

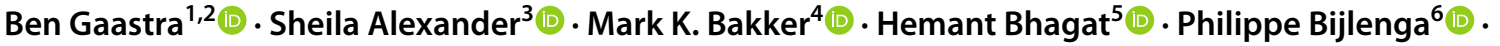

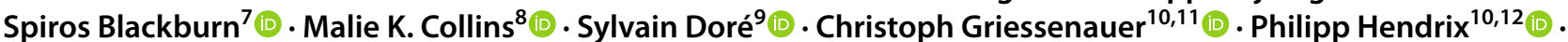

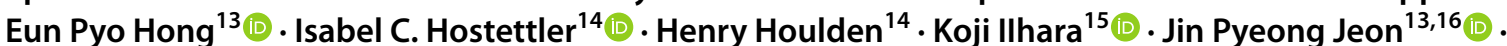

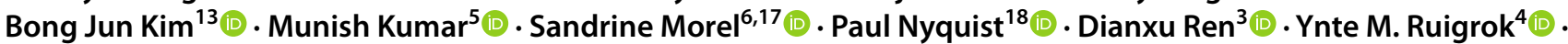

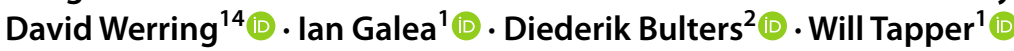

1 Clinical Neurosciences, Clinical and Experimental Sciences, Faculty of Medicine, University of Southampton, Southampton SO17 1BJ, UK

2 Department of Neurosurgery, Wessex Neurological Centre, University Hospital Southampton, Southampton SO16 6YD, UK

3 School of Nursing, University of Pittsburgh, 3500 Victoria Street, Pittsburgh, PA 15261, USA

4 Department of Neurology, University Medical Center Utrecht Brain Center, University Medical Center Utrecht, Heidelberlaan 100, 3584, CX, Utrecht, the Netherlands

5 Division of Neuroanaesthesia, Department of Anaesthesia and Intensive Care, Postgraduate Institute of Medical Education and Research (PGIMER), Chandigarh, India

6 Neurosurgery Division, Department of Clinical Neurosciences, Faculty of Medicine, Geneva University Hospitals, Geneva, Switzerland

7 University of Texas Houston Health Science Center, Houston, TX, USA

8 Geisinger Commonwealth School of Medicine, Scranton, PA, USA

9 Departments of Anesthesiology, Neurology, Psychiatry, Pharmaceutics, and Neuroscience, College of Medicine, Center for Translational Research in Neurodegenerative
Disease, McKnight Brain Institute, University of Florida, Gainesville, FL, USA

10 Department of Neurosurgery, Geisinger, Danville, PA, USA

11 Department of Neurosurgery, Christian-Doppler Klinik, Paracelsus Medical University, Salzburg, Austria

12 Department of Neurosurgery, Saarland University Medical Center, Homburg, Germany

13 Institute of New Frontier Research, Hallym University College of Medicine, Chuncheon, South Korea

14 Stroke Research Centre, University College London, Institute of Neurology, London, UK

15 National Cerebral and Cardiovascular Center Hospital, 6-1 Kishibe-Shimmachi, Suita, Osaka, Japan

16 Department of Neurosurgery, Hallym University College of Medicine, Chuncheon, South Korea

17 Department of Pathology and Immunology, Faculty of Medicine, University of Geneva, Geneva, Switzerland

18 Departments of Neurology, Anesthesia/Critical Care Medicine, Neurosurgery and General Internal Medicine, Johns Hopkins School of Medicine, Baltimore, MD 21287, USA 